\title{
The Rehabilitation of Edentulous Maxilla with the Use of All-on-Four Implants
}

\author{
Adnan Ege Köseler*1, Serkan Sarıdağ', Fatih Mehmet Coşkunses ${ }^{2}$ \\ ${ }^{1}$ Department of Prosthodontics, Kocaeli University, Turkey \\ ${ }^{2}$ Department of Oral and Maxillofacial Surgery, Kocaeli University, Turkey
}

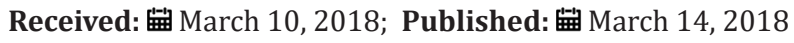

*Corresponding author: Adnan Ege Köseler, Department of Prosthodontics, Kocaeli University, Turkey

\begin{abstract}
Although many approaches have been developed in the rehabilitation of severely atrophic maxilla and mandibles with implantsupported restorations, most of these treatments are expensive and complex. But the All-on-Four concept is an exception which uses only four implants to support an acrylic, screw-retained provisional prosthesis delivered on the day of implant placement, followed by a definitive prosthesis three months later. The All-on-four concept is one such treatment procedure which may be used in case of complete edentulism. This concept is much better than the routine treatment alternative of conventional dentures and has been reported to have successful outcomes in short term, long term and retrospective studies that have been performed in the past.
\end{abstract}

Keywords: Zirconia; All-on-four concept; Implant; Screw; Fixed prosthesis

\section{Introduction}

To replace missing teeth due to the need of esthetics and function, many different treatment modalities are available today. One such treatment is the rehabilitation of empty spaces with dental implants $[1,2]$. Post extraction patients with edentulism or longtime full denture wearers often desire fixed prosthesis. However, these patients often present with minimal posterior bone support. Anatomical structures, namely, the maxillary sinuses in the maxilla and the inferior alveolar nerve in the mandible prevent implant placement in the posterior regions. Because of the resorption following teeth loss, performing a dental implant therapy would not be possible therefore, advanced surgical procedures are often necessary [3]. For an excessively atrophic maxilla, the placement of implant supported fixed restorations necessitate advanced surgical procedures such as sinus lifting and augmentation techniques [4].

This situation, not only increases the treatment time but also is more expensive because of the use of bone grafts. The All-onfour concept eliminates these surgical procedures and provides an opportunity to perform fixed implant supported fixed restorations by using only four implants [4]. This method advocates tilting the distal implants in the edentulous arches which enables the placement of longer implants, improved prosthetic support with shorter cantilever arm, improved inter implant distance and improved anchorage in the bone [5]. Moreover, screw-retained provisional prosthesis can be delivered to the patient on the day of implant placement [6]. The All-on-four concept is an accepted treatment option with high success rates. Several clinical studies have reported high survival rates for tilted implants [7-9]. The concept enables immediate function and esthetics via fixed prosthetic option with good biomechanical properties and the ease of cleaning [10]. It also helps to reduce bone loss, to restore oral functions of both speaking and chewing to minimize the curing time, to cause less pain and discomfort and to improve the patient's self-confidence and quality of life [11].

However, this concept has some limitations that patients must not only have good general health and acceptable oral hygiene but also sufficient bone availability for implants of at least 10 $\mathrm{mm}$ residual bone. Moreover, immediate function can only be established in case of sufficient primary stability of the implants. The All-on-four concept is very technique sensitive and requires elaborate pre-surgical preparation such as CAD/CAM and surgical splints [12]. Because of this, the surgical placement of implants would not always be possible as implant placement is completely prosthetically driven and also the length of the cantilever would 
not be extended as desired. On the other hand, the technique is relatively easy to perform in any patient setting by an experienced surgeon and eliminates the need for advanced techniques.

The reported complications of this concept can be categorized as mechanical, biological, functional and esthetic. A fracture or loosening of mechanical and prosthetic components would be the mechanical complications encountered. Biological complications reported are fistula formation, infection, soft-tissue inflammation and loss of implant stability. Functional complications reported are patient phonetics, cheek and lip biting, chewing ability (based on assessment of the complaints of the patient) and prosthesis retention. Lastly, the esthetic complications reported include hard and soft tissue esthetics [13]. The aim of this report is to present a case of All-on-four concept performed in maxilla and the pros and cons of this concept.

\section{Case Presentation}

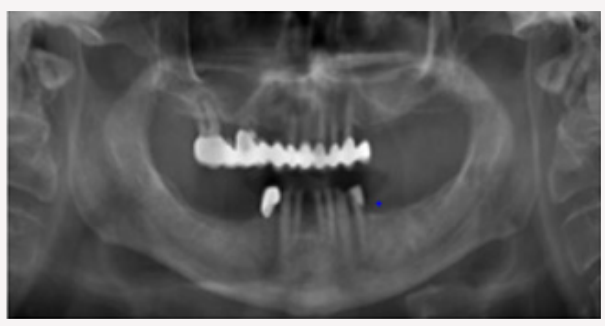

Figure 1: A panoramic view of the atrophic maxilla.

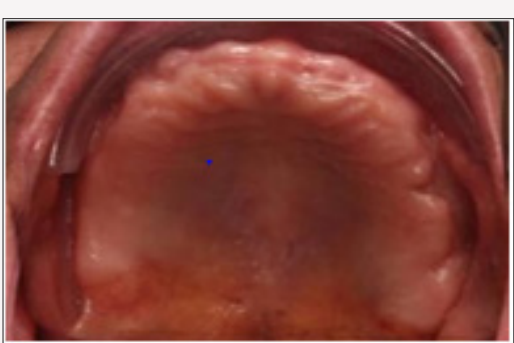

Figure 2: A view of the maxilla after extraction.

A 73 years old non-smoking male patient was referred to our clinic with the complaint of esthetics and function related with severe atrophy of the maxilla. Study models were made and duplicated. The working models were mounted and articulated. Facial photographs were taken after establishing a maximum smile line. Radiographs of the severely atrophic maxilla were taken (Figure 1). The remaining teeth of the maxilla were extracted because of severe periodontitis and bone loss (Figure 2). On evaluation with computed tomography we noticed that the upper posterior segments were severely resorbed and bilateral maxillary sinus lift was necessary. On the mandible between the first premolar teeth, an 8-unit anterior zirconia restoration was planned (Figures $3 \& 4$ ). For the replacement of missing posterior teeth, four Nobel Biocare (Nobel BiocareAB, Göteborg, Sweden) implants were planned on both sides of the jaw with respect to the areas of teeth 35-36 and 4546. In order to establish a fixed prosthesis in the maxilla, two distal tilted implants were placed parallel to the anterior sinus wall with a $30^{\circ}$ angulation relative to the occlusal plane (Figures $5 \& 6$ ). The two mesial sites were prepared at the level of the canines. The insertion axis and the length of the implants were planned on the 3D-CBCT of the patient. The angulation of the distal implants enabled distal abutments, which could emerge in the second premolar area with a great reduction of the distal cantilever. Bone density was assessed by the surgeon during the early phase of drilling with subjective evaluation by using a $2.0-\mathrm{mm}$ drill. The tilted implant sites were underprepared to maximize implant stability. A torque controller with a torque limit of $50 \mathrm{~N} . \mathrm{cm}$ was used during implant placement, and a manual wrench was used in case of incomplete seating of the implant and so primary stability was established as $50 \mathrm{~N} . \mathrm{cm}$ at least.

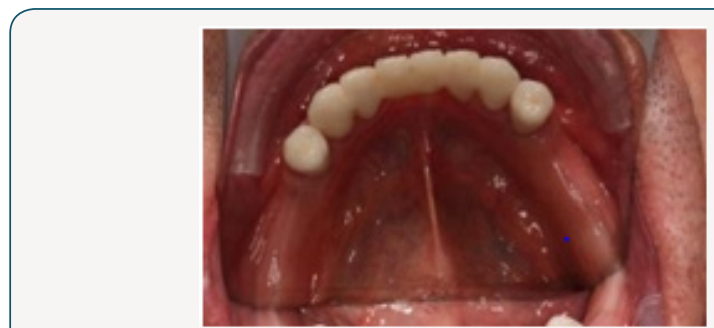

Figure 3: Occlusal view of 8-unit fixed zirconia restorations on the mandible.

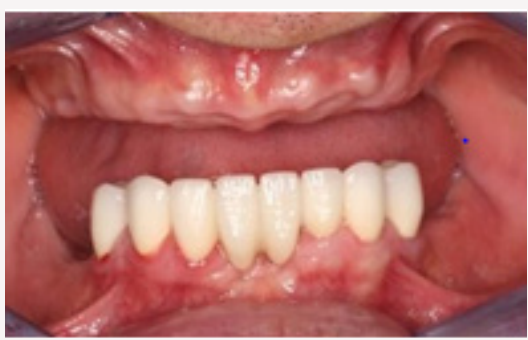

Figure 4: A labial view of zirconia restorations.

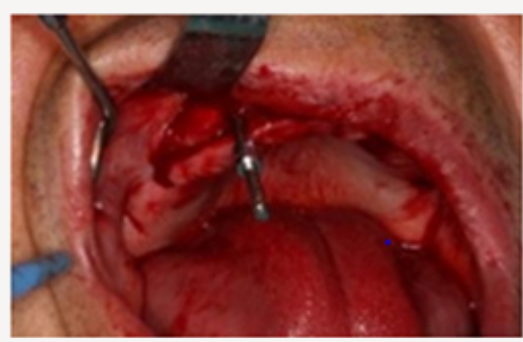

Figure 5: The insertion of distal implant.

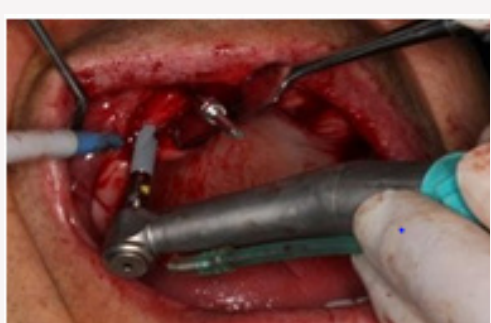

Figure 6: The insertion of distal implant. 
Multi unit abutments (MUA, Nobel Biocare) (Figure 7) and standard straight temporary abutments were connected to the distal implants to allow for an optimal prosthetic screw access, whereas straight abutments (Figure 7) were placed over the mesial implants. Waterproof wound closure was performed and a standard antibiotics regimen was prescribed. The patient was instructed to avoid brushing and any trauma to the surgical site, and cold food was suggested for the first day and a soft diet for the first week thereafter. On the day of surgery, for the temporary prosthesis, holes were drilled through the pre-made previous immediate dentures to identify the positions of the temporary abutments (Figure 8). The holes were enlarged so that the denture can be properly positioned in the mouth. The maxillary denture was first relined and fitted through the over denture abutments with the mid-line aligned and the palate fully seated.

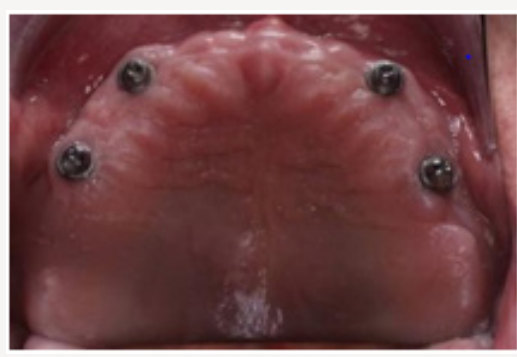

Figure 7: Straight and angled abutments.

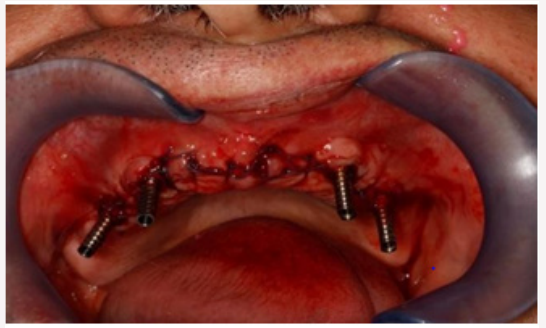

Figure 8: Temporary abutments.

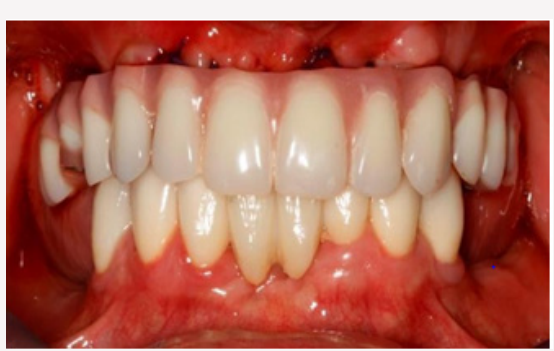

Figure 9: Temporary prostheses.

After the reline acrylic was fully cured, the denture was removed by unscrewing the over denture abutments. The palate of the denture was removed to transform the denture into an acrylic bridge. The tissue side of the bridge was made convex and polished. The bridge was then reattached back to the implant abutments in the mouth. The occlusion was adjusted to canine-guided occlusion
(Figure 9). The patient returned after 6 weeks, and the maxillary bridge was removed in order to review soft tissue healing. After 3 months, definitive prosthesis construction was performed. The abutments were fixed with dental floss and rigidly splinted with flowable composite resin material (Figure 10). In order to decrease the tension between the splinted abutments, the link between the left and right side was cut.

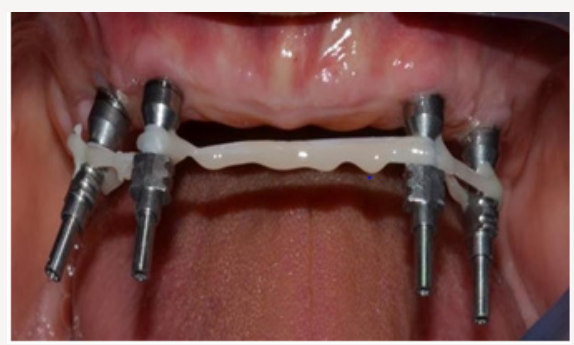

Figure 10: Rigid splinting.

An individualized acyrlic tray was prepared to fit properly and allow stress-free movement of the abutments. With the help of polyether impression material, a definitive impression was made (Figure 11). The vertical length, mid-line, smile-line (Figure 12) and occlusion were checked and after the control of the metal framework (Figure 13), definitive prosthesis was adjusted with canine-guided occlusion and luted with polycarboxylate cement (Figure 14). A final panoramic image was taken (Figure 15). After 2-years follow-up, all the restorations were stable and there were no signs of peri-implant bone loss which is considered as a good treatment outcome.

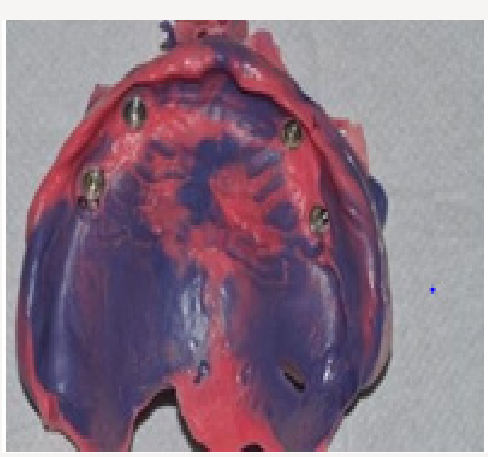

Figure 11: Polyether impression made with individualized acrylic tray.

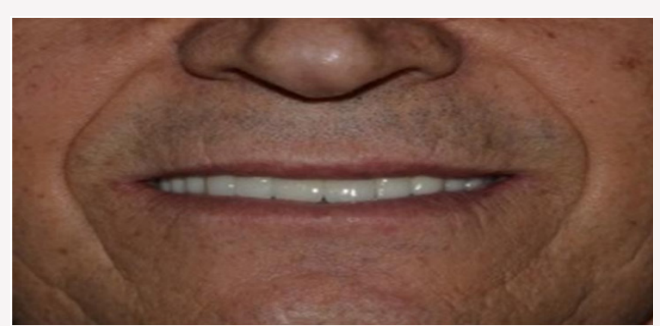

Figure 12: Smile-line and mid-line evaluation. 


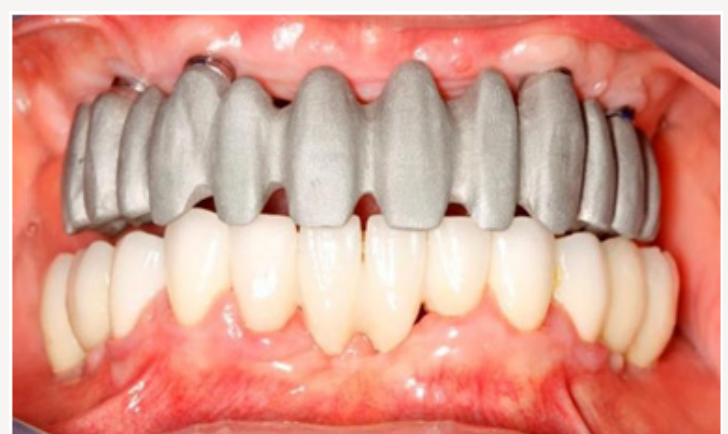

Figure 13: Metal framework.

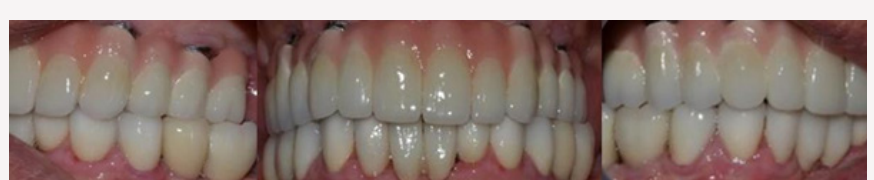

Figure 14: Occlusion.

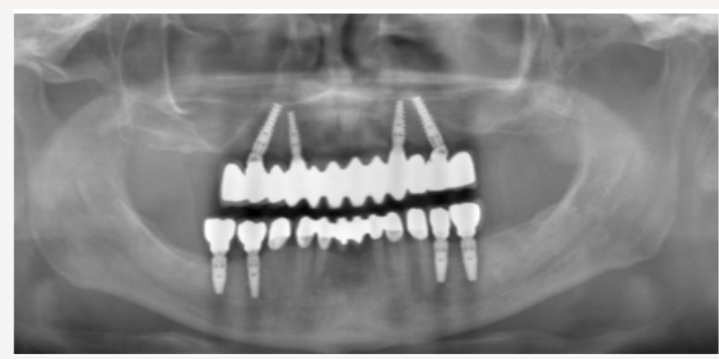

Figure 15: Final panoramic image.

\section{Discussion}

The All-on-four treatment concept may not be considered as a conventional treatment modality by some clinicians. However, this treatment concept uses four implants to support a fixed prosthesis without advanced surgical techniques and is extremely attractive for most patients and clinicians [1,2]. Malo and colleagues reported $97.6 \%$ 1-year survival rate with the 128 branemark implants in 32 patients. Additionally, Malo and colleagues reported $97.2 \%$ 1-year survival rate for mandible and 100\% 1-year survival rate for maxilla with 92 Nobel Speedy implants in 23 patients. After 9 years of follow-up, Malo and colleagues reported $96.2 \%$ survival rate, and after up to 5 years follow-up for maxilla $97.7 \%$ survival rate achieved [14].

Effectively, the cumulative success rate of implants placed for the complete rehabilitation of the maxilla ranged between $93 \%, 4 \%$ and $100 \%$ for early loading with a follow-up between 1 and 5 years [15-18], and between $91 \%$ and $100 \%$ for immediate function with a follow-up between 1 and 5 years [19-23]. The present treatment concept uses the load- bearing capacity of the maxillary bone in a favourable manner. Owing to the freedom of tilting, the implants can be anchored in dense bone structures (anterior bone with higher density) and well-spread anteriorly-posteriorly, giving an effective prosthetic base. By reducing the number of implants to four, each implant can be placed without interference with adjacent implants [24]. This treatment approach, using tilting and few implants rather than inserting several implants competing for space, has demonstrated good results in a previous study with delayed loading [3], and in immediate function [19].

In this case, we rigidly splinted our implants by fixing them with dental floss and flowable composite. Underestimating the stress that would occur between the four implants would be a mistake as it would affect the final impression and prevent the passive fit of the prosthesis, which is a 'must' for the All-on-four concept. Therefore, in this case, we cut the connection of the four implants and lowered the stress to ensure a passive fit. To provide immediate functioning, all-acrylic prosthesis were placed a few hours after the surgery. These prostheses are usually used as provisional prostheses for restoring esthetics and function. But most of time clinicians encounter the risks of fracture. These failures can be related to careless design and manufacturing processes, inadequate implant support or occlusion [25,26]. It is important to have a canine-guided occlusion and posterior disclusion in order to decrease cantilever forces around the implants.

The use of tilted implants is recommended in the cases of maxillary atrophy [13]. The main advantages of this technique, when compared to bone graft or zygomatic implant techniques are lesser surgical morbidity as well as optimal use of the residual alveolar ridge. Furthermore, there are no significant differences between axial and tilted implants in terms of success rates and marginal bone loss [24,27]. Implant angulations has many advantages, including increased implant length, apical cortical fixation, and secondary stabilization from the sides of the implants and, most importantly, increased anteroposterior spread [28-30] and decreased length of cantilevers. The most important biomechanical requirements for the immediate function in the highly atrophic maxilla are adequate anteroposterior spread, defined as $12-15 \mathrm{~mm}$ and cantilever length. Cantilevers should not be present in the provisional prosthesis but can be approximately $10 \mathrm{~mm}$ in a bar-supported definitive prosthesis. This in turn can significantly decrease the vertical forces on the implants as well as the interfacial stresses and strains.

Implants placed in the maxilla without angulation but with good anteroposterior spread can be short and relatively unstable, usually requiring a delayed placement strategy. The placement of implants in a region of denser bone tissue allows for lesser total treatment time, lesser cost and avoidance of multiple surgeries $[31,32]$. As a conclusion based on reviewed studies, it is suggested that immediate loading associated with tilted implants could be considered to be a viable therapeutic option for the atrophic maxilla and that there does not seem to be a different clinical outcome between tilted and axial implants. 


\section{References}

1. Khatami AH, Smith CR (2008) All-on-four immediate function concept clinical report of treatment of an edentolous mandible with a fixed complete denture and milled titanium framework. J Prosthodont 17(1): 47-51.

2. Agliard E, Panigatti S, Clerico M, Villa C, Malo P (2010) Immediate rehabilitation of the edentulous jaws with full fixed prostheses supported by four implants: Interim results of a single cohort prospective study. Clinical Oral Implants Research 21(5): 459-465.

3. Fortin Y, Sullivan RM, Rangert BR (2002) The Marius implant bridge: surgical and prosthetic rehabilitation for the completely edentulous upper jaw with moderate to severe resorption: a 5-year retrospective clinical study. Clinical Implant Dentistry and Related Research 4(2): 6977.

4. Malo P, Nobre M, Petersson U, Wigren S (2006) A pilot study of complete edentulous rehabilitation with immediate function using a new implant design: case series. Clinical Implant Dentistry and Related Research 8(4): 223-232.

5. Aparicio C, Perales P, Rangert B (2001) Tilted implants as an alternative to maxillary sinus grafting: a clinical, radiologic, and periotest study. Clinical Implant Dentistry and Related Research 3(1): 39-49.

6. Attard NJ, Zarb GA (2005) Immediate and early implant loading protocols: a literature review of clinical studies. The Journal of Prosthetic Dentistry 94(3): 242-258.

7. Maló P, Rangert B, Nobre M (2005) All-on-4 immediate-function concept with Brånemark System implants for completely edentulous maxillae: A 1-year retrospective clinical study. Clinical Implant Dentistry and Related Research 7(1): 88-94.

8. Capelli M, Zuffetti F, Del Fabbro M, Testori T (2007) Immediate rehabilitation of the completely edentulous jaw with fixed prostheses supported either upright or tilted implants: A multicenter clinical study. The International Journal of Oral \& Maxillofacial Implants 22(4): 639644.

9. Testori T, Del Fabbro M, Capelli M, Zuffetti F, FrancettiL, et al. (2008) Immediate occlusal loading and tilted implants forthe rehabilitation of the atrophic edentulous maxilla. 1-year interim results of a multicenter prospective study. Clinical Oral Implants Research 19(3): 227-232.

10. Christopher CK (2012) Implant rehabilitation in the edentulous jaw. The "All-on-4" immediate function concept. Aust Dent Pract: 138-148.

11. Malo P (2013) All-on-4 Treatment Concept for total edentulous rehabilitation- safely, securely, affordably and fast. Aust Dent Pract: 148.

12. M Taruna, B Chittaranjan, N Sudheer, Suchita T, MD Abusaad (2014) Prosthodontic Perspective to All-On-4® Concept for Dental Implants. Journal of Clinical \& Diagnostic Research 8(10): 16-19.

13. Malo P, Nobre M, Lopes A, Francischone C, Rigolizzo M (2011) Allon-4 immediate- function concept for completely edentulous maxillae: A clinical report on the medıum (3 years) and long-term (5 years) outcomes. Clinical Implant Dentistry \& Related Research 14(1): 139150.

14. Charles AB, Gary TK, John B (2012) The All-On-Four Immediate Function Treatment Concept With Nobel Active Implants: A Retrospective Study. The Journal of Oral Implantology 37(4): 437- 443.

15. Olsson M, Urde G, Andersen E, Sennerby L (2003) Early loading of maxillary fixed cross-arch dental prostheses supported by six or eight oxidized titanium implants: results after 1 year of loading, case series. Clinical Implant Dentistry \& Related Research 5(Suppl 1): 81-87.

16. Fischer K, Stenberg T (2004) Early loading of ITI implants supporting a maxillary full- arch prosthesis: 1-year data of a prospective, randomized study. The International Journal of Oral \& Maxillofacial Implants 19(3):
374-381.

17. Ostman PO, Hellman M, Sennerby L (2005) Direct implant loading in the edentulous maxilla using a bone density-adapted surgical protocol and primary implant stability criteria for inclusion. Clinical Implant Dentistry \& Related Reserach 7(Suppl 1): S60-69.

18. Fischer K, Stenberg T (2006) Three-year data from a randomized, controlled study of early loading of single-stage dental implants supporting maxillary full-arch prostheses. Int J Oral Maxillofac Implants 21(2): 245-252.

19. Tarnow DP, Emtiaz S, Classi A (1997) Immediate loading of threaded implants at stage 1 surgery in edentulous arches: Ten consecutive case reports with 1- to 5-year data. Int J Oral Maxillofac Implants 12(3): 319324.

20. Grunder U (2001) Immediate functional loading of immediate implants in edentulous arches: two-year results. Int J Periodontics Restorative Dent 21(6): 545-551.

21. Rocci A, Martignoni M, Gottlow J (2003) Immediate loading in the maxilla using flapless surgery, implants placed in predetermined positions, and prefabricated provisional restorations. A restrospective 3-year clinical study. Clin Implant Dent Relat Res 5(Suppl 1): 29-36.

22. Balshi SF, Wolfinger GJ, Balshi TJ (2005) A prospective study of immediate functional loading, followingthe Teeth in a Day protocol: a case series of 55 consecutive edentulous maxillas. Clin Implant Dent Relat Res 7(1): 24-31.

23. Degidi M, Perrotti V, Piattelli A (2006) Immediately loaded titanium implants with a porous anodized surface with at least 36 months of follow-up. Clin Implant Dent Relat Res 8(4): 169-177.

24. Krekmanov L, Kahn M, Rangert B, Lindstrom H (2000) Tilting of posterior mandibular and maxillary implants of improved prosthesis support. Int J Oral Maxillofac Implants 15(3): 405-414.

25. Wolfinger JG, Balshi JT, Rangert B (2003) Immediate functional loading of Brånemark System implants in edentulous mandibles: clinical report of the results of developmental and simplified protocols. Int J Oral Maxillofac Implants 18(2): 250-257.

26. Nikellis I, Levi A, Nicolopoulos C (2004) Immediate loading of 190 endosseous dental implants: a prospective observational study of 40 patient treatments with up to 2-year data. Int J Oral Maxillofac Implants 19(1): 116-123.

27. Takahashi T, Shimamura I, Sakurai K (2010) Influence of number and inclination angle of implants on stress distribution in mandibular cortical bone with All-on-4 Concept. J Prosthodont Res 54(4): 179-184.

28. Brunski J (2003) Biomechanics in Osseointegration in Dentistry. Quintessence 2: 49-83.

29. Jensen OT, Adams MW, Smith E (2012) Paranasalbone: the prime factor affecting the decision to use transsinus vs. zygomatic implants for biomechanical support for immediate function in maxillary dental implant reconstruction. Oral and Craniofac Tissue Eng 2: 198-206.

30. Parel SM, Phillips WR (2011) A risk assessment treatment planning protocol for the four implant immediately loaded maxilla: preliminary findings. J Prosthet Dent 106(6): 359-366.

31. Graves S, Mahler BA, Javid B, Armellini D, Jensen OT (2011) Maxillary all on four therapy using angled implants: a 16 month clinical study of 1110 implants in 276 jaws. Dent Clin North Am 55(4): 779-794.

32. Cricchio G, Sennerby L, Lundgren S (2011) Sinus bone formation and implant survival after sinus membrane elevation and implant placement: a 1-6 year follow-up study. Clin Oral Implant Res 22(10): 1200-1212. 
This work is licensed under Creative Commons Attribution 4.0 License

To Submit Your Article Click Here:

Submit Article

DOI: 10.32474/MADOHC.2018.01.000119

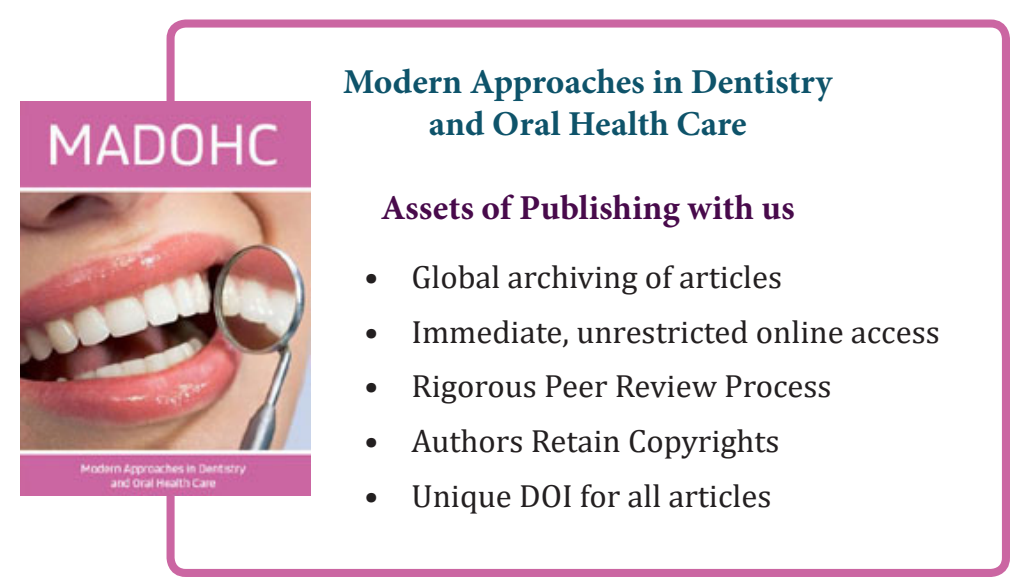

